Johannes Illenberger, Gunnar Flötteröd, Kai Nagel

\title{
Enhancing MATSim with capabilities of within-day re-planning
}

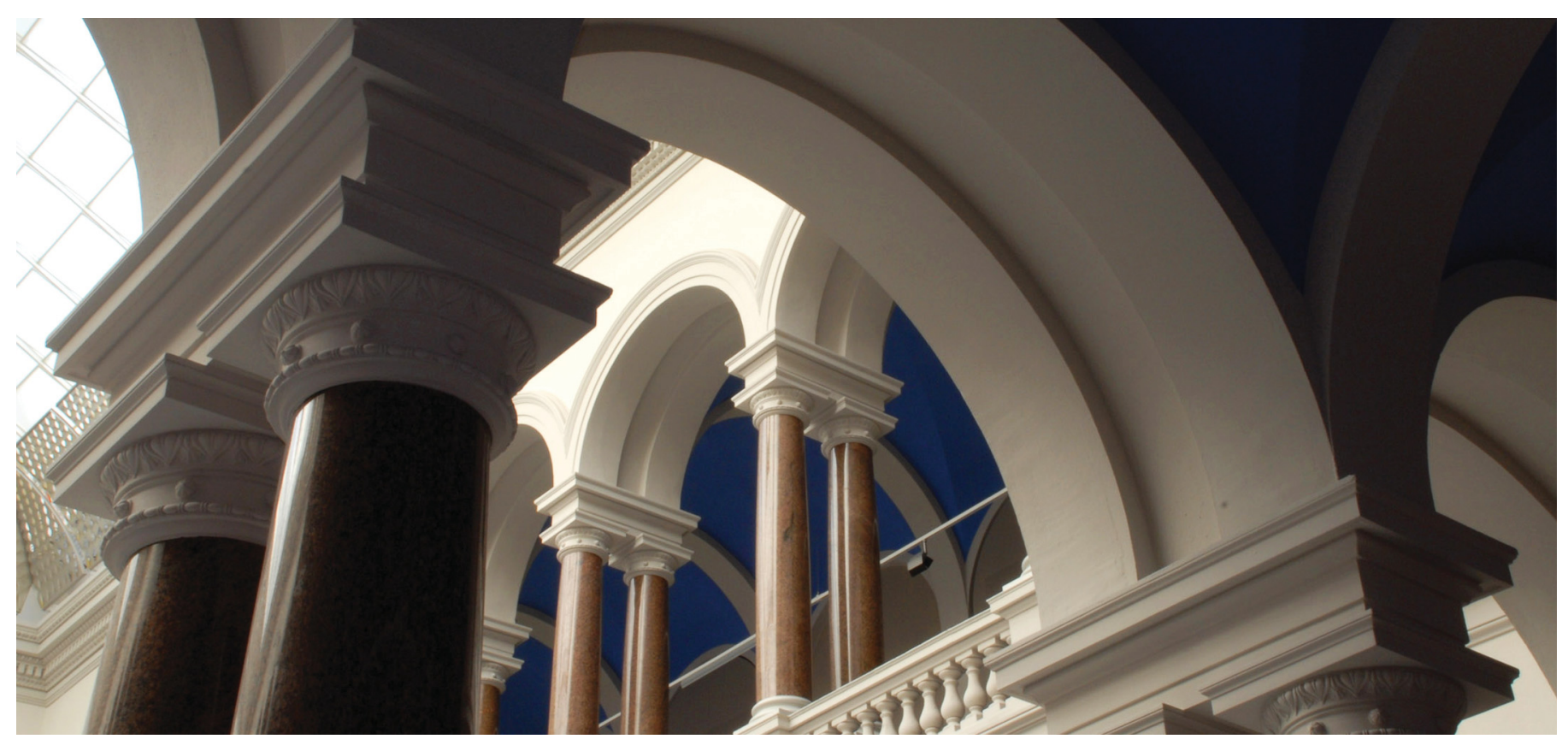

Illenberger, J.; Flötteröd, G.; Nagel, K. (2007). Enhancing MATSim with capabilities of within-day replanning. 2007 IEEE Intelligent Transportation Systems Conference. https://doi.org/10.1109/ITSC.2007.4357810 


\title{
Enhancing MATSim with capabilities of within-day re-planning
}

\author{
Johannes Illenberger ${ }^{1}$, Gunnar Flötteröd ${ }^{2}$, Kai Nagel ${ }^{3}$
}

\begin{abstract}
This paper presents a framework for simulation of within-day re-planning for the MATSim project. Three major building blocks are presented, each of which represents specific aspects of driver behavior. These components comprise (i) the provision of descriptive information in the form of link travel costs, (ii) prescriptive information in the form of routes, and (iii) a model of driver satisfaction. An exemplary model is presented, which focuses on en-route re-planning under different types of information provision. In this model driver perception is constrained to link traversal costs and decisions are made by application of a standard shortest path algorithm. The satisfaction of a traveler is modeled with a scoring (utility) function that evaluates routes as well as activities travelers are aiming at. The framework's applicability is tested with a simple fictive network and a real-world network of Greater Berlin.
\end{abstract}

\section{INTRODUCTION}

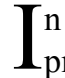
the field of transport planning, engineers agree that the problems of transportation are no more a matter of extending the infrastructure with concrete and steel, but rather a matter of the efficient use of existing transport networks [1]. Advanced Traveler Information Systems (ATIS) are intended to fill in here by providing accurate information through a variety of devices.

An important aspect is the response of drivers to provided information. Since deployment of ATIS technologies is still in an early state, practical experiences are limited. To gain more insights into travelers' decision making in-laboratory experiments such as FASTCARS ([2] and [3]) and IGOR [4] have been proposed.

Behavioral models derived from the results of these laboratory experiments can be used in large-scale simulations to evaluate ATIS technologies. Travel time savings have been observed in several studies ([5], [6], [7] and [8]), varying from three to 30 percent depending on market penetration and network topology. Beside the lowering of travel time itself, the reduction of its uncertainty deserves to receive just as much attention.

As a contribution to the research in this field, this paper presents a basic framework that enhances the MATSim toolkit ("Multi-Agent Transport Simulation Toolkit", www.matsim.org) by capabilities of within-day and en-route re-planning. The framework is integrated in the so-called „mental layer" of agent behavior and defines a set of

\footnotetext{
${ }^{1}$ Johannes Illenberger is with the group for Transport Systems Planning and Transport Telematics, Technische Universität Berlin, D-10587 Berlin, Germany, mail: illenberger@vsp.tu-berlin.de.

${ }^{2}$ Gunnar Flötteröd is with the group for Transport Systems Planning and Transport Telematics, mail: floetteroed@vsp.tu-berlin.de.

${ }^{3}$ Kai Nagel is head of the group for Transport Systems Planning and Transport Telematics, mail: nagel@vsp.tu-berlin.de.
}

interfaces in which specific implementations can model specific behavioral patterns. In detail, the behavior of an agent is represented by modules, each of which reflects a certain behavioral aspect. The modularity allows to easily exchange certain building blocks or even complete behavioral implementations and thus to compare and evaluate different models.

The framework allows to model ATIS strategies, corresponding driver reactions, and finally to analyze the interaction between ATIS, drivers and traffic conditions.

The remainder of this article is organized as follows: In section II, a short introduction to MATSim is given and in section III, the abstract agent model and its conceptual background are presented. Section IV describes an exemplary model and its implementation and section $\mathrm{V}$ verifies the frameworks applicability by means of two scenarios. The paper closes with a discussion and an outlook in section VI.

\section{MATSIM OVERVIEW}

MATSim is a multi-agent based transport simulation which originally envolved from TRANSIMS [9] and pursues an activity-based approach to demand generation. Unlike other transportation simulation packages MATSim is throughout agent-based and generates individual activity plans as input to the network loading rather than (timedependent) origin-destination matrices as typically used in dynamic traffic assignment. More details about the demand generation in MATSim can be found in [10] and [11].

Specifically, a plan contains the agent's intended schedule of activities for the day, and the travel legs connecting the activities. A leg holds several attributes describing the travel from one activity to another such as departure time, expected arrival time, route and transportation mode. Activities contain type attributes such as home, work, education, leisure as well as further information regarding activity timing.

The initial plans are generated by disaggregating census data. Next, there is a mechanism that allows the agents to learn and optimize their plans. The system iterates between plan generation (the mental layer, also referred as strategic layer) and traffic flow simulation (the physical layer). The system remembers several plans per agent and scores the performance of each plan with a fitness function. Between two iterations agents are able to modify plans with the use of genetic algorithms. Those plans are modified by mutation and recombination, e.g. recalculating new routes or varying departure times, while "bad" plan instances are eventually discarded. 
This day-to-day re-planning mechanism, or more generally period-to-period re-planning since a plan must not necessarily be constrained to one day, is continued until the plans are "relaxed", i.e. in an approximate user equilibrium.

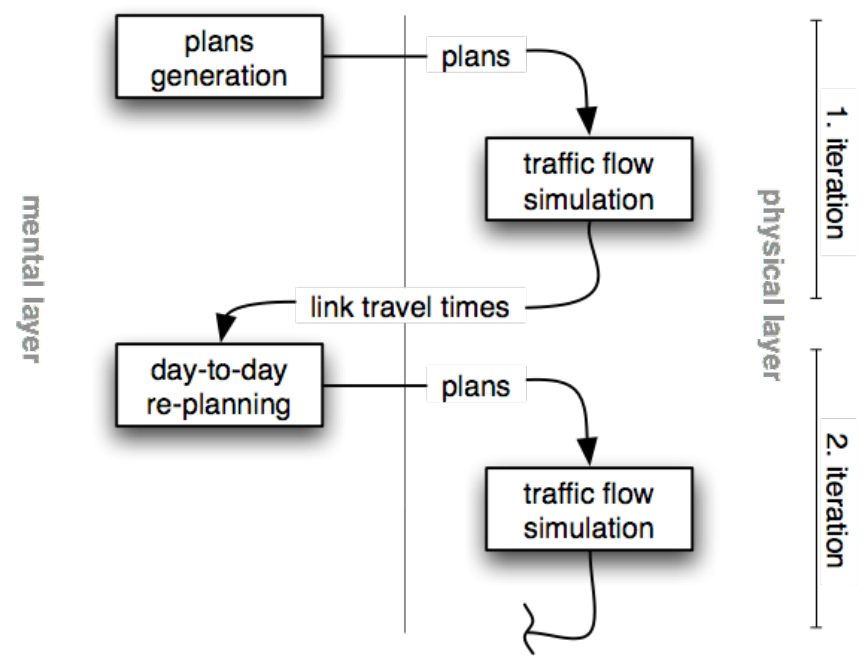

Fig. 1. The system iterates between plan generation/modification and traffic flow simulation until an approximate user equilibrium is reached.

The framework proposed in this paper enhances MATSim with capability of within-day re-planning. More specifically, agents are now not only able to adapt plans between one execution in the traffic flow simulation, but also to modify them during the traffic flow simulation. This enables the agents to spontaneously react to unforeseeable incidents during a day. Plans, which are modified during the traffic flow simulation can now be regarded as a new mutated instance for the plans generation and day-to-day learning process, although such a post-processing has not yet been investigated.

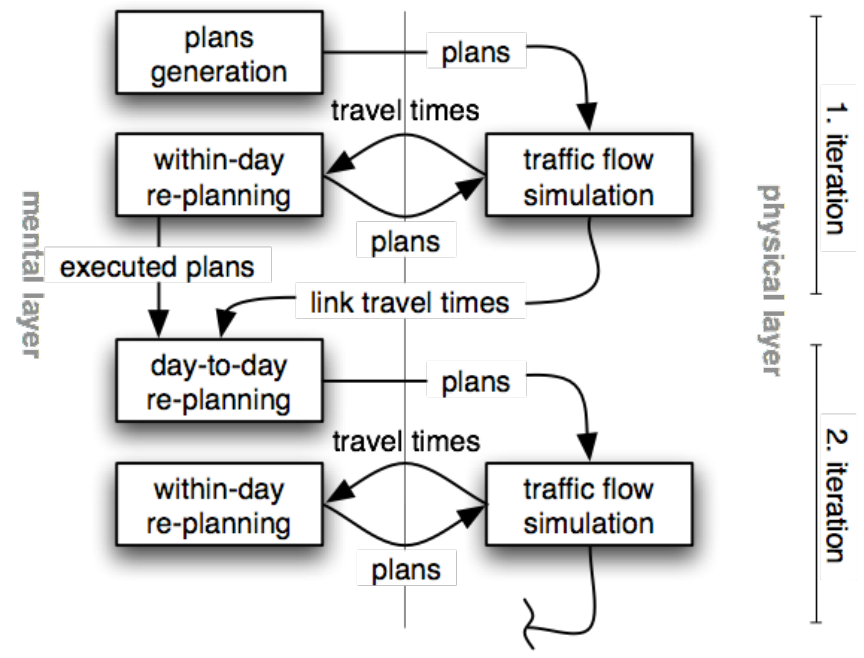

Fig. 2. The traffic flow simulation is enhanced with within-day re-planning capabilities. Agents are now able to mutate their plans during the run of the traffic flow simulation.

Technically, day-to-day and within-day re-planning are quite similar. The agent's capabilities about how it can modify its plan are the same and will be discussed later. However, there are differences considering the knowledge the agent possesses. With day-to-day re-planning the agent acts based on the knowledge it accumulated over previous runs of the traffic flow simulation. Whereas with within-day replanning the agent accesses rather locally available information, such as current link travel times, and thus can react to unforeseeable fluctuations in the traffic system.

To clearly distinguish between the packages of MATSim, the package for plans generation and day-to-day re-planning (represented by the box "plans generation" and "day-to-day replanning" in fig. 2) will be referred as the demand-modeling package and the part that enhances the within-day replanning capabilities (represented by the box "within-day replanning" in fig. 2) will be referred as the telematics package. For the purpose of this paper the telematics package will be used as a standalone system without the feedback of mutated plans to the demand-modeling package depicted in fig. 2.

The following section describes the abstract agent model that enables the basic within-day re-planning capabilities.

\section{ABSTRACT MODEL DESCRIPTION}

\section{A. Basic agent model}

The behavior of an agent is fully determined through its plan. The linkage between the mobility simulation in the physical layer and the mental agent representation is done by the so called basic agent class. The basic agent holds an activity plan and extracts the information required by the mobility simulation out this plan. In particular these are: (i) departure time, (ii) departure link, (iii) destination link and (iv) the agent's desired next link (if the agent is en-route). Altering an agent's behavior is possible only by modification of its plan.

\section{B. Agent brain}

Presented so far, agent behavior is constrained to execution of a predefined activity plan. In order to alter its behavior it needs to become intelligent. Intelligence is provided to the agent by equipping it with a brain object. The essential task of a brain is to do on the fly modification to the plan. Additionally, the brain is able to determine the agent's desire to re-plan at all. Possible capabilities of a brain are departure time choice, activity location choice, variations in activity sequencing and route choice.

An agent brain comprises three further components, each of which represents a certain aspect of the re-planning process. Descriptive information in the form of link travel costs are provided by a so called link cost provider, prescriptive information in the form of predefined routes are obtained from a route provider and the agent's satisfaction is modeled by the contentment module. However, the ways in which these three components are combined are up to a concrete implementation of an agent brain. Figure 3 provides an overview. 


\section{The re-planning process}

On can interpret the activity plan as the agent's intention and the link cost provider as the agent's believes. However, the abstract model does not specify any commitment rules which defines when to re-plan. Also the agent's desires (maximizing utility, maintaining the plan, etc.) are not defined and are to be specified in a particular model and its implementation.

The re-planning mechanism as it is processed by the agent brain can be separated in three quite typical steps [12]:

1. Perception. The agent observes its current environment which basically is given by the current traffic state or retains information out if its memory. The sensor system through which the agent perceives or accesses its memory are represented by the link cost providers.

2. Deliberation. The agent follows a certain strategy to fulfill its desires. Both, the strategy and the desires are to be specified in a particular model implementation. Note that the deliberation about to re-plan or not has already been done prior to these steps by a certain commitment rule. Once the re-planning process has been triggered the agent definitely wishes to do so.

3. Execution. The agent modifies its plan according to its deliberation in the previous step so that the mobility simulation will move it through the network following its new plan.

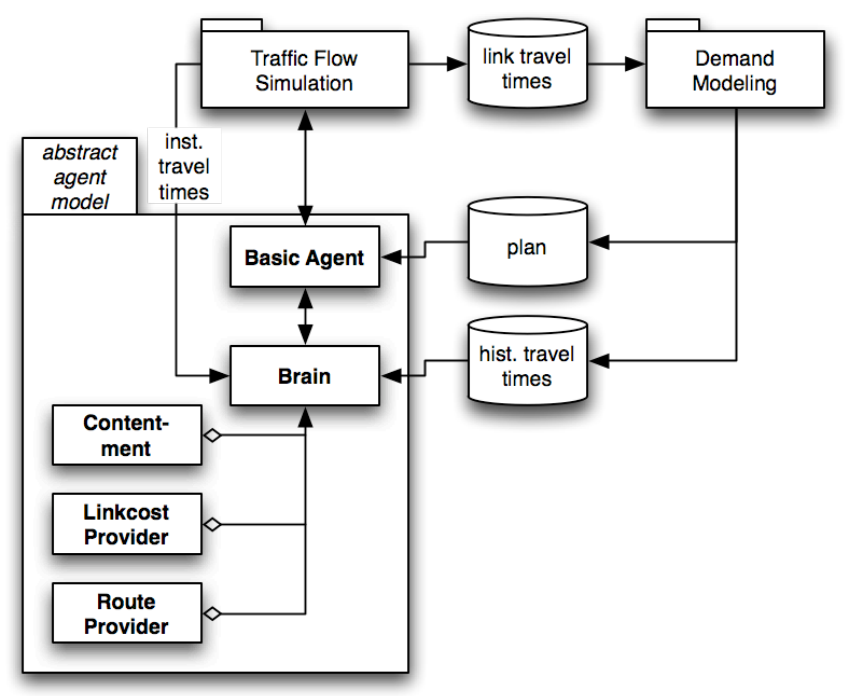

Fig. 3. Abstract agent model architecture. An agent is fed with an activity plan generated by the demand-modeling package. The plan can be modified by the brain with the help of a link cost provider, route provider and contentment module. The mobility simulation moves the agent through the network according to its plan. Information about instantaneous link travel times are provided by the traffic flow simulation. Additionally the brain can access the knowledge the agent accumulated in previous iterations.

\section{Types of information provision}

As mentioned above, the link cost provider and the route provider represent two types of information sources. From the conceptual side one can distinguish between intrinsic and extrinsic provision. However, technically these sources of information are dealt with in a unified way.

An intrinsic link cost provider may represent the agent's observation (what it can see by looking out of the window) or the historical knowledge an agent accumulated in previous trips. An extrinsic link cost provider may represent an in-vehicle device, which supplies the driver with travel times or messages broadcasted via radio.

For route providers this differentiation is not as distinct as above. One may imagine an intrinsic route provider as a representation of the process of acquiring a route when the agent thinks by itself. The extrinsic counterpart may be an in-vehicle navigation device, internet-based services, variable destination signs (VDS) or even static guide posts.

The simulation framework currently implemented provides three different types of basic link travel time information.

Historical travel times represent the ,typical“ state of the traffic network ${ }^{4}$ as expected by the traveler, reactive travel times (in literature also referred as naïve or instantaneous travel times) represent a current snapshot of the traffic network, and predictive travel times represent a forecast of the traffic state within a given time window. ${ }^{5}$

Depending on the type of link travel costs that are supposed to be modeled, an implementation of a link cost provider may compose the three basic types to a new representation of link costs. Of course link cost providers are not restricted to the information generated by the mobility simulation. Static information may be read out of files or generated by other modules a priori. It would be even conceivable that a link cost provider operates its own subsimulation to generate specific information.

\section{EXEMPLARY MODEL AND IMPLEMENTATION}

This section presents an exemplary model implementation. Its purpose is to validate the frameworks applicability rather than to model realistic travelers' behaviour.

\section{A. Model assumptions}

The model focuses on route switching, i.e. re-planning is only done en-route with modifications to the agent's current route.

The agents' desire is to always maintain the timing of its plan, only being early is not considered as undesirable. The contentment module is now used as a part of a commitment rule, which decides when to re-plan. If the agent notices that it will be late (e.g. due to congestion) it becomes displeased and whishes to re-plan. To maintain its plan, the agent tries

\footnotetext{
${ }^{4}$ Historical travel times are the accumulated knowledge of previous iterations. As the relaxation process advances travel times are getting close to a user equilibrium.

${ }^{5}$ Such a prediction is generated once for all agents in a rolling-horizon manner by running the mobility simulation forward without re-planning and then switching back to the previously marked state.
} 
to find a faster route based on its believes of link costs. However, its available information is based on individual observations and estimation of its surroundings and thus is limited by the extent of its perceptivity.

The re-planning process is triggered as follows: Agents are asked at each intersection by the simulation controller about their desire to re-plan. If an agent is displeased the replanning process is triggered. However, re-planning is computationally rather expensive (due to route searching) and thus the simulation controller does only allow a certain fraction of agents to re-plan. As the agent's desire to re-plan increases the probability that the controller selects it increases, too. I.e., the simulation controller always tries to determine the fraction of agents in such a way that it will select the most displeased agents.

In this case the simulation logic diverts from a truly agentorientated approach, since a traveller that does feel the need to re-plan would definitely do so in the real world. Truly agent-oriented implementations would use independent computing threads for each agent, and these threads might decide by themselves when they become active. Current computers are, however, not able to process as many threads as agents (approx. 200,000 agents for large scenarios). Systems with much more lightweight micro-threads might be a way out and are a topic of ongoing research [13].

\section{B. Link cost perception}

An agent has two ways to acquire information: It can observe its direct surroundings and it can estimate information that cannot directly be perceived. Estimation is based on current observation in combination with historical and common knowledge. Observations are based on the current state of the traffic network but are distorted by individual errors of perception. Consequently, to model the driver's perception and estimation of link travel times the historical and reactive travel times are required.

Observations are spatially limited to the agent's current link and on its immediately succeeding links (i.e. all outgoing links of its downstream node). For these links the agent is aware of the reactive (instantaneous) travel times. However, to create behavioral diversity in a simple way the agent's perception is distorted by two individual perception errors. The first is a white noise added to the reactive travel times while the second represents the uncertainty of appraising the correct travel time when the current traffic state differs from the habitual known. If, e.g., link travel times are twice as high as historically observed, an agent is more unsure about the real travel time than if observations match its historical knowledge.

Travel times of unobservable links are scaled according to the agent's current observation of link travel times compared to the historically learned. Additionally, scaled and perceived link travel times are corrected if they fall below the free flow travel time.

\section{Routing}

The routing is done with a time variant Dijkstra best path algorithm. The routing algorithm is supplied with link travel costs by a link cost provider as described in the previous section B.

In the literature, route choice models are often realized as random utility models that account for the non-deterministic behavior of humans [14]. However, MATSim focuses on large-scale scenarios, and a discrete choice model for route choice appears quite expensive in terms of computational performance. Thus we choose a purely simulation-based approach, apply strict shortest path algorithms, and realize the non-deterministic behavior by randomization of link costs as described in the previous section.

\section{Contentment}

Our exemplary implementation of the agent brain uses a contentment module to determine the agent's need to re-plan. Contentment is represented as a scalar value out of the interval $[-1,1]$, where 1 means the agent is pleased, 0 the agent is indifferent, and -1 the agent is displeased. As an agent becomes displeased (i.e. values less than 0), its need to re-plan increases.

The implementation for the contentment module used here is based on a scalar scoring (utility) function for plans introduced by Charypar and Nagel in [11]. This scoring function evaluates the quality of a plan by summing the utilities of all activities that are performed and all travel (dis)utilities.

The utility for performing an activity is a logarithmic function of activity duration whereas the penalty, or more precise the negative utility for travel is modeled as a linear function of trip duration.

The contentment of an agent is defined as the quotient of the expected plan score (based on the agent's current beliefs of travel times) and the initial plan score (calculated from the demand-modeling package).

\section{Simulation}

\section{A. Introduction}

To validated the applicability of the proposed framework, two scenarios with the above introduced model were run which will be described in the following: first a synthetic corridor example (called the "simple" scenario), and then a scenario based on real-world data from Berlin (called the "Berlin" scenario). For both scenarios, the set-up is as follows:

- As base case, a set of initial plans is given. Plans contain departure times and routes for every agent.

- These plans are then run through the simulation described in this paper. A number of experiments have been conducted where the fraction of agents that are allowed to re-plan has been varied. This set of agents is chosen according to both, a global re-planning probability and the agents' need to re-plan. This is done in such a way, that agents with high re-planning needs are preferred for re-planning.

For the "simple" scenario, the initial plans are manually constructed. For the "Berlin" scenario, they are taken from 
the MATSIM demand-modeling package. The plans for the "Berlin" scenario can be considered relaxed, i.e. approximately in an user equilibrium. However, since the equilibrium is only approximate agents are still able to somewhat improve their performance.

\section{B. "Simple” test scenario}

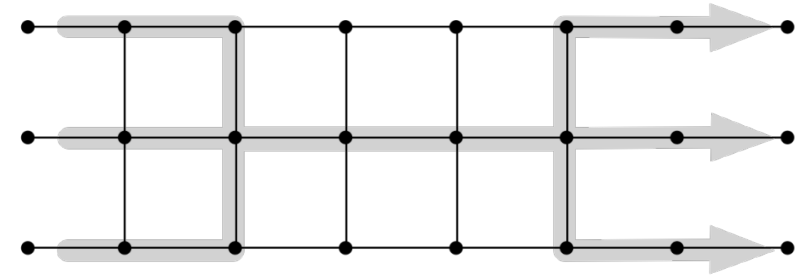

Fig. 4. Simple test network. The gray arrows denote the routes as defined in the initial plans. According to their initial plans agents depart at the six leftmost horizontal links and travel via the middle route to the three rightmost links.

We first set up a simple test scenario with a grid shaped network including 41 links. All links are equal in their attributes (1000 m length, 1800 vehicles per hour max. flow and $7.5 \mathrm{~m} / \mathrm{s}$ free speed). The demand consists of 6000 agents departing at 7:00 and traveling from the left to the right side (see fig. 4).

According to their initial plans, all agents use the middle route. Because of its limited capacity, spillback occurs shortly behind the demand entry points. The resulting travel times are used as the historical traffic pattern. It is questionable if this is realistic since the historical travel times are not in user equilibrium. However, these simulation runs are to demonstrate the capabilities of the presented framework and rather than to simulate realistic travel behaviour. Furthermore this extreme case shows more clearly the effects of within-day re-planning.

We run several simulations with different types of link cost providers to investigate the impact of descriptive information provision. Beside the model of agents' link travel time perception presented in IV.B, we additionally provided the agents directly with historical, reactive and predictive travel times. As a comparison criterion we use the average deviation from user equilibrium, i.e. the difference of the route's duration the agents actually experienced and the best route calculated a posteriori, and averaged this over all agents. In the "simple" scenario the average deviation is 9:50 for a simulation run without within-day re-planning.

Figure 5 shows results that one may not expect at first glance. Predictive information provision leads to less deviation from a user optimum than reactive information provision. But re-planning with historical travel times leads to better results than with reactive (instantaneous) travel times. The link cost provider which models the agent's perception produces results that are between the ones of the historical and the reactive link cost provider. At this point the cause of the peak in the graph for historical travel times is unknown.

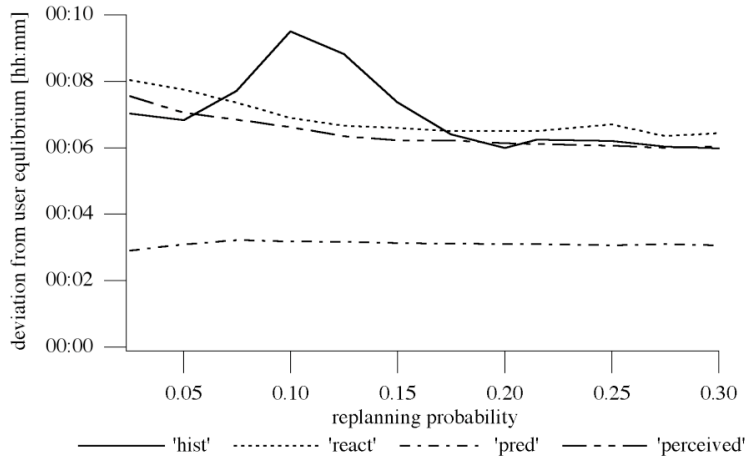

Fig. 5. Comparison of link cost provider in the simple test scenario. 'hist' = historical, 'react' $=$ reactive, 'pred' = predictive (with 30 mins prediction window) and 'perceived' = perceived travel times.

The results with reactive information provision demonstrate the problem of overreaction. Overreaction describes the situation in which drivers overcompensate in response to information, again causing sub-optimal traffic conditions. This effect can be well observed with high replanning probabilities.

\section{C. "Berlin" test scenario}

To investigate the applicability of the framework in real world applications, a large-scale scenario with a reduced road network representing the metropolitan area of Berlin (Germany) has been set up. The network includes approximately 2400 links and is bounded by the Berlin beltway (fig. 6).

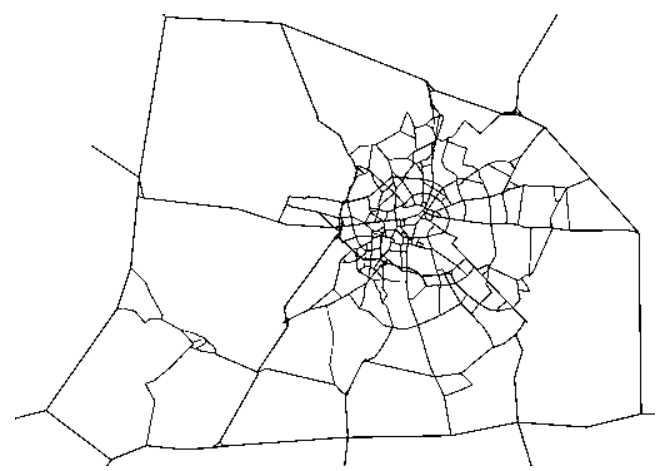

Fig. 6. Reduced road network representing the metropolitan area of Berlin.

Activity plans are now taken form the MATSim demandmodeling package and represent a 10 percent sample of Berlin's population (approx. 170,000 agents).

The same investigations as with the simple test scenario have been conducted for the Berlin scenario. The average user equilibrium deviation without re-planning is 5:06.

On qualitative inspection of fig. 7 we now observe the expected results: Better information leads to better results ("predictive" < "reactive" < "perceived" < "historical"). The phenomenon of overreaction with reactive information provision does not occur in this scenario.

But in contrast to the simple scenario, increasing replanning probability does not always decrease the equilibrium deviation. With the use of historical or perceived 
travel times it even dramatically impairs results. Recall that the initial plans for the Berlin scenario have undergone several iterations in the MATSim demand-modeling package and are thus close to the user equilibrium. Accordingly, it is not possible to significantly improve the traffic state by providing additional information to drivers and as more agents are allowed to divert from their original route the traffic state moves further away from the equilibrium.

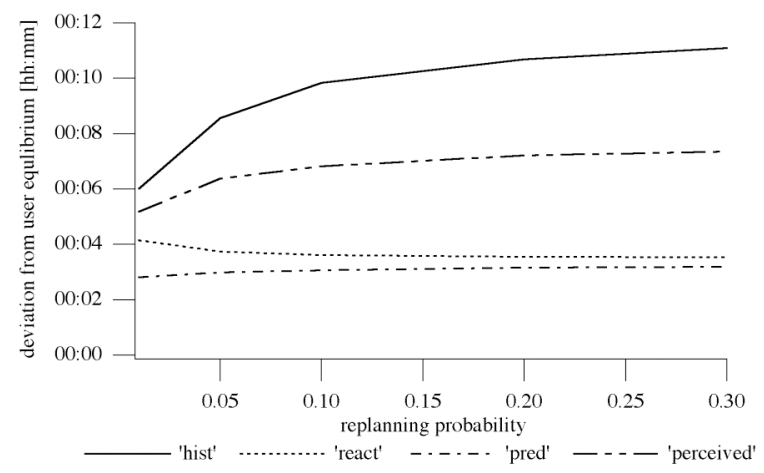

Fig. 7. Comparison of link cost provider in the Berlin test scenario. 'hist' $=$ historical, 'react' $=$ reactive, 'pred' $=$ predictive $($ with 30 mins prediction window) and 'perceived' = perceived travel times.

Considering the results of the predictive information provider, the increasing values of user equilibrium deviation seem to be related to the accuracy of the prediction. For remembrance, the prediction is done by running the simulation forward without within-day re-planning. It is obvious that if more agents are allowed to re-plan, the experienced traffic state potentially differs more from the prediction as if fewer agents are allowed to re-plan.

\section{CONCLUSION}

This work presented an agent-based framework to enhance MATSim with capability of within-day re-planning. The abstract model distinguishes between a module for link cost perception, route searching and contentment. Together, these modules represent an agent's behavior. The framework provides flexible options for adjusting the behavior by choosing different implementations of the modules. For the particular model presented here, Dijkstra's best pathalgorithm has been used for the route searching model, a scoring function to model the contentment, and historical, reactive, predictive travel times as well as combinations of them to model the perception of link costs. Beside the exemplary implementation, additional implementations modeling simple within-day destination choice [15] and guidance by means of variable message signs [16] exist in this within-day re-planning framework.

Two test scenarios demonstrated the frameworks applicability. Although the exemplary model does not claim to mimic realistic behaviour the simulation results appear to be reasonable. Of course, further applications will require a more careful calibration and validation of the model.

Altogether, it can be expected that the presented framework provides valuable insights into the effects of ITS measures not only in current and future traffic conditions, but also on driver contentment itself.

Our future research will concentrate the decision making process considering travel time uncertainty and risk aversion. In this context it will be practicable to also deal with departure time choice which has been neglected in our early studies.

\section{REFERENCES}

[1] G. Wolfgang Heinze. Kurskorrektur - Eine Ortsbestimmunt der Raumordnung aus Verkehrssicht. Working Paper 06-6, Inst. for Land and Sea Transport Systems, TU Berlin, Germany, 2006.

[2] Adler, J.L., Recker, W.W., and McNally, M.G. "A Conflict Model And Interactive Simulator (FASTCARS) for Predicting En-route Driver Behavior in Response to Real-Time Traffic Condition Information". Transportation 20 (2), 83-106, 1993.

[3] Adler, J.L, Recker, W.W., and McNally, M.G. "Using Interactive Simulation to Model Driver Behavior under ATIS". Proceedings of the ASCE 4th International Conference on Microcomupters in Transportation, Baltimore, MD, 1992.

[4] Bonsall, P.W. and Parry, T. "Using an Interactive Route-Choice Simulator to Investigate Drivers' Compliance with Route Guidance Advice". Transportation Research Record 1306, pp 59-68, 1991.

[5] Adler, J. L., V. J. Blue \& T. L. Wu. "Assessing Network and Driver Benefits From Bi-Objective In-vehicle Route Guidance", presented in the 78th TRB Annual Meeting, TRB, 1999.

[6] Emmerink, R. H. M., K. W. Axhausen, \& P. Rietveld. "Effects of Information in road transport networks with recurrent congestion", Transportation 22, pp.21-53, 1995.

[7] Wunderlich, K. E. "An Assessment of Pre-Trip and en route ATIS Benefits in a Simulated Regional Urban Network", in the 3rd world Congress on Intelligent Transport Systems, Intelligent Transportation: Realizing the Future, Orlando, Florida, 1996.

[8] Levinson, D., Gillen, D., Chang, E. "Assessing the Benefits and Costs of Intelligent Tranportation Systems: The Value of Advanced Traveler Information Systems", California PATH Research Report, UCB-ITSPRR-99-20, 1999.

[9] TRANSIMS http://transims.tsasa.lanl.gov/. TRansportation ANalysis and SIMulation System, accessed 2007. Los Alamos National Laboratory, Los Alamos, NM.

[10] B. Raney, K. Nagel; An improved framework for large-scale multiagent simulations of travel behavior; in: P. Rietveld, B. Jourquin and K. Westin (eds.), Towards better performing European Transportation Systems.

[11] D. Charypar and K. Nagel. Generating complete all-day activity plans with genetic algorithms. Transportation, 32(4):369-397, 2005.

[12] J. Ferber. Multi-agent systems. An Introduction to distributed artificial intelligence. Addison-Wesley, 1999.

[13] L. Bläser. A Component Language for Structured Parallel Programming, JMLC 2006, LNCS 4228 Springer 2006.

[14] M. Ben-Akiva and M. Bierlaire. Discrete choice methods and their applications to short-term travel decisions. Kluwer, 1999.

[15] Gunnar Flötteröd and Kai Nagel. Bayesian modeling and estimation of combined route and activity location choice. Working Paper 06-3, Inst. for Land and Sea Transport Systems, TU Berlin, Germany, 2006.

[16] Carl Rommel. Automatic Feedback Control Applied to microscopically Simulated Traffic - The potential of route guidance in the Berlin traffic network. Master's thesis, Inst. for Land and Sea Transport Systems, TU Berlin, Germany, 2007. 